increased the mean percentage of apoptotic cells $(47.8 \pm 4.8 \%$ vs $9.3 \pm 1.2 \%$ in control cells, $p<0.05)$. A cDNA microarray study showed significant $(p<0.05)$ upregulation of interleukin (IL)-1 B-convertase, caspase-3, -8 and -9 genes were confirmed in real-time RT-PCR in cHSP60 and cHSP10 stimulated than in control cells. The transcript levels of IL-1ß and IL-18 in cells treated with cHSP60 and cHSP10 was found to be significantly $(p<0.05)$ higher in stimulated than in control cells.

Conclusions In women with persistent chlamydial infection, the release of extracellular cHSPs may lead to cell apoptosis and to an inflammatory response involved in fibrosis and scarring of female genital tract that may contribute severe tubal pathologies including infertility.

\section{P4-S1.02 COUPLING OF ELECTROCHEMICAL DETECTION WITH PCR AMPLIFICATION FOR SENSITIVE DETECTION OF NEISSERIA GONORRHOEAE}

doi:10.1136/sextrans-2011-050108.504

${ }^{1} \mathrm{~S}$ Sood, ${ }^{1} \mathrm{R}$ Verma, ${ }^{2} \mathrm{R}$ Singh, ${ }^{2} \mathrm{G}$ Sumana, ${ }^{3,4} \mathrm{~V} \mathrm{~K}$ Sharma, ${ }^{1} \mathrm{~J} \mathrm{C}$ Samataray, ${ }^{5} \mathrm{R}$ M Pandey, ${ }^{2} \mathrm{~B}$ D Malhotra. ${ }^{1}$ Department of Microbiology, All India Institute of Medical Sciences, New Delhi, India; ${ }^{2}$ Biomolecular Electronics and Conducting Polymers Research Group, National Physical Laboratory, New Delhi, India; ${ }^{3}$ Department of Dermatology, All India Institute of Medical Sciences, New Delhi, India; ${ }^{4}$ Department of Venerology, All India Institute of Medical Sciences, New Delhi, India; ${ }^{5}$ Department of Biostatistics, All India Institute of Medical Sciences, New Delhi, India

Background Despite the recent development of different detection methods, better diagnostic tools are required for quick and reliable detection of pathogens. Use of biosensors for detection of pathogens is gradually gaining momentum. However, PCR amplification of DNA target is still necessary for application on biosensor for accurate detection of the pathogen. An in-house PCR using self-designed primers targeting the opa gene (GenBank accession no. PUID 9716120 SNUM $2706 \mathrm{Ng}$ _opa) was performed. The generated amplicons were used to evaluate the DNA biosensor utilising a 19mer oligonucleotide sequence (GenBank PUID SNUM: 9716119 $2705 \mathrm{Ng}$ opa) as probe.

Methods In-house PCR was standardised and an amplified product (amplicons) of 188 bps were obtained for positive samples. Fabrication of bioelectrode was performed by immobilising the activated probe onto pre-treated screen-printed gold electrodes. The fabricated nucleic acid functionalised gold electrode was characterised using, SEM, CV, DPV techniques. The presence of target DNA was detected electrochemically by monitoring the redox peak of methylene blue indicator. Standardisation of working conditions was done using complementary, non-complementary, one base mismatch DNA, and amplicons from standard strain of N. gonorrhoeae (ATCC 49226) \& 16 clinical isolates. In addition, DPV measurements of hybridised bioelectrode with amplicons of 26 clinical samples of which 10 were culture positive, was done. A cutoff value for positives was determined by using the software STATA (version 9)

Results The analytical sensitivity of PCR was $10-17 \mathrm{M}$ of DNA and the bioelectrode could detect up to $1.0 \times 10-20 \mathrm{M}$ of the DNA amplicons. An $11.49 \%$ decrease in signal intensity was taken as the cut-off. Samples giving an equivalent or more decrease than this value were considered as positives.

Conclusions The coupling of electrochemical detection with PCR amplification showed the advantage of higher sensitivity and increased specificity for detection of $N$ gonorrhoeae. This may prove to be particularly valuable for the identification of asymptomatic infections and could greatly improve gonorrhoea control.

\section{P4-S1.03 DEMONSTRATING PERFORMANCE OF A LOW-COST, ULTRA-RAPID PCR DEVICE WITH TRUE POINT-OF-CARE APPLICATIONS}

doi:10.1136/sextrans-2011-050108.505

${ }^{1} \mathrm{D}$ Pearce, ${ }^{1} \mathrm{D}$ Shenton, ${ }^{1} \mathrm{~S}$ Brake, ${ }^{1} \mathrm{~A}$ Larry, ${ }^{1} \mathrm{M}$ Green, ${ }^{2} \mathrm{C}$ Gaydos. ${ }^{1}$ Atlas Genetics Ltd., Trowbridge, UK; ${ }^{2}$ Johns Hopkins University, School of Medicine, Baltimore, USA

Background We are developing a highly sensitive, ultra-rapid, multiplex PCR method with fully-integrated DNA preparation and ambient-stable reagents. The assay was used in conjunction with a novel electrochemical detection method to demonstrate low copy number amplification and detection in $<20 \mathrm{~min}$, as a point-of-care (POC) diagnostic test for Chlamydia trachomatis (CT).

Methods The method employs custom PCR cards, utilising a thinfilm laminate construction to achieve rapid heat transfer, in conjunction with an ultra-rapid thermocycler. All reagents necessary to perform the extraction, amplification and detection are deposited into the cards and air dried at the point of manufacture. Novel, ambient-stable reagent formulations with an 18 month shelf life have been developed. A sample is added to the card and DNA extracted from the sample. The resulting eluate reconstitutes dried PCR reagents and a 40-cycle multiplex PCR is performed using rapid thermocycling. Amplified target is detected using electrochemically-labelled target-specific probes and a doublestranded DNA-specific exonuclease to release the electrochemical label. Released label is read by applying a voltage to a screen printed carbon electrode and at a known oxidation potential the label is oxidised producing a measurable current. The unique rapid performance of this device has been demonstrated in terms of analytical sensitivity and reagent stability under ambient storage conditions. Multiplex capability is demonstrated in this test with the presence of internal control (IC) DNA.

Results Analytical sensitivity of the device was evaluated by testing dilutions of CT in the presence of IC DNA. The results show CT detection down to 50 copies when co-extracted, amplified in duplex and detected electrochemically with the IC DNA (see graph). Tests on the reagents dried into the device showed stability for 18 months when stored at ambient temperature $\left(20-25^{\circ} \mathrm{C}\right)$. Reagent performance after 18 months' storage was shown to be equivalent to performance at time zero see Abstract P4-S1.03 figure 1.

Conclusions The results show that this device could be used to perform ultra-rapid multiplex PCR with no user intervention after sample addition, allowing minimally-trained staff to carry out the assay in $<20 \mathrm{~min}$, meeting the needs for a "true" POC device. Ambient stability of the reagents negates the requirement for any specialised storage conditions.

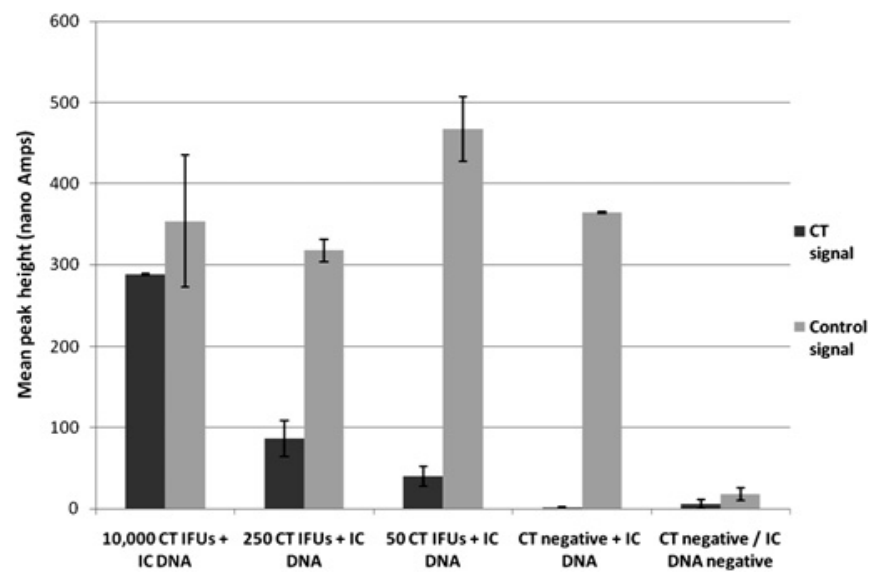

Abstract P4-S1.03 Figure 1 